\title{
A System Based Modelling Approach for Anatomic Joints
}

\author{
R. Leskovar* A. Körner* F. Breitenecker* \\ * Technische Universität Wien, Institute for Analysis and Scientific \\ Computing, Vienna, Austria (e-mail: \{ruth.leskovar, andreas.koerner, \\ felix.breitenecker\}@tuwien.ac.at).
}

Keywords: Multibody Modelling, System Simulation, Biomechanics, Human Joints, Feedback Control.

\section{INTRODUCTION AND STATE OF THE ART}

This contribution deals with the development of a system based modelling approach for anatomic joints. Modelling and simulation in the field of biomechanics is a very important method to analyse the dynamic behaviour of the human body. Understanding the influence of the individual parts of the musculoskeletal system is necessary for the analysis and treatment of diseases. Furthermore, mathematical models of parts of the human body are indispensable in the development of prostheses.

The engineering progress in the last decades in the field of prostheses lead to an enormous enhancement of life quality for disabled people. Nowadays, wearing a prosthesis does not restrict daily life and normal habits so much. Modern leg prostheses adapt to the gait cycle by changing the damping of the knee as humans do it automatically. This technology reduces the risk of falling and extends activities with prostheses as doing sports and walking on uneven ground. Sensoring the knee angle and ground contact of the heel gives the possibility to control the change of the swung into the stance phase and vice versa via a microprocessor in the prosthesis. For example this technology is used in prostheses developed by OttoBock ${ }^{\circledR}$ (2011), a well known manufacturer of healthcare systems.

Among several methods exist to model a biomechanical system, two approaches to describe these systems are most common:

- Partial differential equations

- Multibody modelling.

Modelling a biomechanical system with partial differential equations is used to compute the strains and stresses in the components during small movements. Multibody models are used to describe the kinematics of the underlying system under gross movements. These two approaches describe the biomechanical system on a microscopic and macroscopic level, respectively. In order to formulate a PDE model a detailed knowledge about the system is required which is not every time available.

The main part of this contribution is the integration of a biomechanical system in a system simulation loop circuit and not the formulation of an additional biomechanical model for a human joint. This gives the opportunity to improve the technology used in prostheses and the research in the field of biomechanics of human joints.

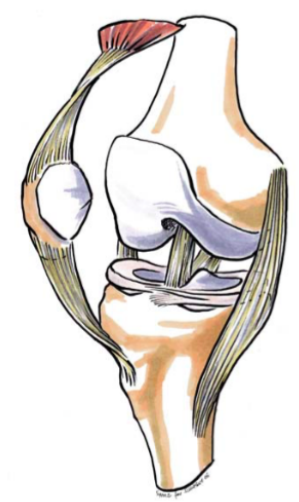

Fig. 1. The anatomy of a human knee joint in Schabus and Bosina (2007).

\section{MULTIBODY MODELS}

Multibody models give the possibility to describe a biomechanical system without having all information about the interactions in the system. The possibility to build a multibody model in an acausal way does not require the mathematical formulation of the system from the first instance on. This does not only allow a fast way of modelling but an analysis of the system. Multibody models thus emphasize the most important elements in a system and their mutual interactions. Hence, multibody models are a powerful tool to model and simulate the kinematics of body parts because it focuses on the mechanical description. A mutibody model contains bodies respresenting flexible or rigid human body parts and links connecting multiple bodies with different types of movements and degrees of freedom. The equations of motion of a multibody model are derived by the Newton-Euler equations and the Lagrange formalism. The motion of rigid bodies can therefore be described by

$$
M \ddot{q}+J_{q}^{T} \lambda=F .
$$

Here $M$ describes the mass matrix of the system, the vector $q$ denotes the system coordinates which contain the translational and rotational movements, the matrix $J_{q}$ is the Jacobian matrix of the system coordinates, $\lambda$ represents the Lagrange multipliers and the vector $F$ implies the external forces. The derivation and use of this formula is explained more detailed in Quental et al. (2012). 


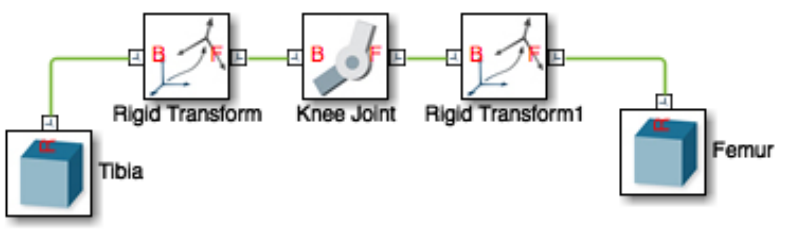

Fig. 2. Simple multibody model of a human knee joint in Simscape.

This description of the dynamics in a human body part by ordinary differential equations allows to consider the biomechanical system as a dynamic system which behaviour changes in time $t$ depending on acting inputs $u$, disturbances and initial values $p$, where the output $y$ can be described with an ordinary differential equation $f$ :

$$
\dot{y}(t)=f(t, u(t), y(t), p) \text {. }
$$

\section{CASE STUDY - THE HUMAN KNEE JOINT}

For the first case study we choose the human knee joint to implement as multibody system. The human knee joint is the most complex joint in the human body due to the complexity of the interactions of three bones and multiple ligaments and tendons. The anatomic structure of the human knee is shown in figure 1 with the three bones, the patella, the femur and the tibia as well as the main ligaments, the cruciate and the collateral ligaments. First we implement a simplified knee model only with the two main bones connected by a revolute joint. The bones are implemented as rigid bodies. The multibody model of this system is developed in Simscape with the multibody model library which can be seen in figure 2 .

\section{INTEGRATION OF A JOINT MODEL IN A SYSTEM SIMULATION LOOP}

As mentioned before the focus of this work is the integration of biomechanical models in a system simulation loop circuit. This closed feedback loop system will be designed in the first attempt for the multibody model but future work will include biomechanical models described by partial differential equations as well and later perhaps additional modelling approaches. This required flexibility of the loop can be seen in figure 3 .

To design a closed loop it is necessary to define the input $u$ and the output $y$ of the model in order to create a closed feedback system. In the case of the knee the input would be an acting force on the bone, e.g. the quadriceps muscle which is activating the femur and via the knee joint the tibia as well. Alternatively, modelling a prosthesis for the whole lower limb, the leading microprocessor exerts the force. In this respect, the output $y$ can be the angle of the knee joint in the simplest case to analyse in which phase of the gait cycle the leg is. The controller which reacts depending on the output of the biomechanical model of the knee, defines the acting force on the knee joint for the next time step. This design of a closed feedback simulation loop gives the opportunity to simulate a human gait. For the first approach it will be a walk on even ground but it is possible to design a more complex controller able to adapt not only to the ground but also to the walking speed.

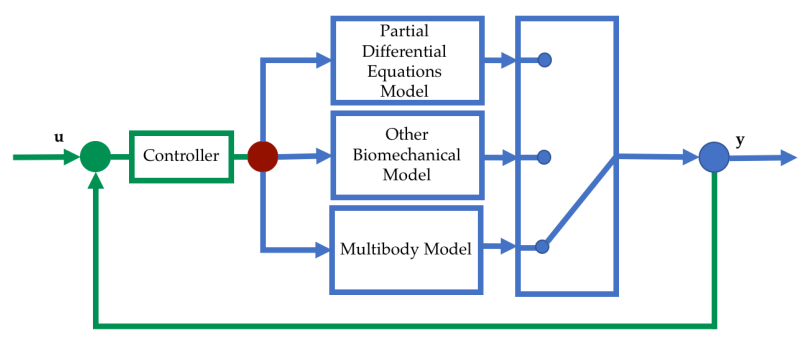

Fig. 3. Design of a feedback loop for different biomechanical models.

To ensure a stable control design specifications for the used model must be made. This will include restrictions for the in- and the output. This work will specialise on models which compute under certain forces the resulting strains in the human knee. This restriction makes sure that the designed controller works for different types of biomechanical models.

In order to design a well defined closed system simulation loop circuit, the system has to depend on time. This means the output of the biomechanical system changes their behaviour not only dependent on the input and parameters but also additionally on time.

\section{OUTLOOK}

Further work will focus on the specification of the biomechanical model, e.g. ligaments and muscles will be included to analyse a more complex system behaviour, related to Bersini et al. (2016). Moreover, the controller designed for the multibody model will be evaluated for different biomechanical models such as thus based on PDEs. The focus will lay on the examination of the performance and efficiency of the feedback simulation loop depending on different models and their complexity. The aim of this work is to investigate the requirements of biomechanical models for different applications which should help to validate them depending on their applications.

After evaluating the closed loop simulation circle designed for the knee joint, different joint models, as for example a shoulder joint, will be examined.

The results of the established work will be beneficial for the future design of biomechanical models and finally in the development of prosthetical products.

\section{REFERENCES}

Bersini, S., Sansone, V., and Frigo, C.A. (2016). A dynamic multibody model of the physiological knee to predict internal loads during movement in gravitational field. Computer Methods in Biomechanics and Biomedical Engineering, 19(5), 571-579. doi: 10.1080/10255842.2015.1051972.

OttoBock ${ }^{\circledR}(2011)$. Genium. Technical report, Otto Bock HealthCare GmbH.

Quental, C., Folgado, J., Ambrósio, J., and Monteiro, J. (2012). A multibody biomechanical model of the upper limb including the shoulder girdle. Multibody System Dynamics, 28(1), 83-108. doi:10.1007/s11044-011-92970 .

Schabus, R. and Bosina, E. (2007). Das Knie - Diagnostik, Therapie und Rehabilitation. Springer-Verlag Wien New York. 